\title{
Long-term Outcome After Monosegmental L4/5 Stabilization for Degenerative Spondylolisthesis With the Dynesys Device
}

\author{
Sven Hoppe, MD, ${ }^{*}+$ Othmar Schwarzenbach, MD, * Emin Aghayev, MD, + Harald Bonel, MD, $\S$ \\ and Ulrich Berlemann, MD*
}

Study Design: Retrospective analysis of prospectively collected clinical data.

Objective: To assess the long-term outcome of patients with monosegmental L4/5 degenerative spondylolisthesis treated with the dynamic Dynesys device.

Summary of Background Data: The Dynesys system has been used as a semirigid, lumbar dorsal pedicular stabilization device since 1994. Good short-term results have been reported, but little is known about the long-term outcome after treatment for degenerative spondylolisthesis at the L4/5 level.

Methods: A total of 39 consecutive patients with symptomatic degenerative lumbar spondylolisthesis at the L4/5 level were treated with bilateral decompression and Dynesys instrumentation. At a mean follow-up of 7.2 years (range, $5.0-11.2 \mathrm{y}$ ), they underwent clinical and radiographic evaluation and quality of life assessment.

Results: At final follow-up, back pain improved in $89 \%$ and leg pain improved in $86 \%$ of patients compared with preoperative status. Eighty-three percent of patients reported global subjective improvement. Ninety-two percent would undergo the surgery again. Eight patients $(21 \%)$ required further surgery because of symptomatic adjacent segment disease (6 cases), lateonset infection ( 1 case), and screw breakage (1 case). In 9 cases, radiologic progression of spondylolisthesis at the operated segment was found. Seventy-four percent of operated segments showed limited flexion-extension range of $<4$ degrees. Adjacent segment pathology, although without clinical correlation, was diagnosed at the L5/S1 (17.9\%) and L3/4 (28.2\%) segments. In 4 cases, asymptomatic screw loosening was observed.

Conclusions: Monosegmental Dynesys instrumentation of degenerative spondylolisthesis at L4/5 shows good long-term

Received for publication March 27, 2012; accepted October 5, 2012.

From the *Das Rückenzentrum, Spinal Surgery, Thun; $\dagger$ Department of Orthopaedic Surgery, University of Bern, Inselspital; §MEM Research Center, Bern; and §Department of Radiology, University of Bern, Inselspital, Bern, Switzerland.

The authors declare no conflict of interest.

Reprints: Sven Hoppe, MD, Department of Orthopaedics and Traumatology, University of Bern, Inselspital, Bern 3010, Switzerland (e-mail: svenhoppe@gmail.com).

Copyright (C) 2012 Wolters Kluwer Health, Inc. All rights reserved. results. The rate of secondary surgeries is comparable to other dorsal instrumentation devices. Residual range of motion in the stabilized segment is reduced, and the rate of radiologic and symptomatic adjacent segment degeneration is low. Patient satisfaction is high. Dynesys stabilization of symptomatic L4/5 degenerative spondylolisthesis is a possible alternative to other stabilization devices.

Key Words: monosegmental degenerative spondylolisthesis, dynamic stabilization, long-term follow-up, Dynesys

(Clin Spine Surg 2016;29:72-77)

$\mathrm{D}$ egenerative lumbar spondylolisthesis (DLS) is a common condition in elderly patients and a frequent cause of spinal stenosis. DLS most commonly occurs at the L4/5 level. Although most patients can be treated conservatively, up to $15 \%$ may require surgical treatment because of refractory back and leg pain, spinal claudication, and/or radicular pain. ${ }^{1}$ Decompression of the spinal stenosis may improve symptoms of claudication and radicular pain, ${ }^{1,2}$ and fusion is intended to prevent progression of the sagittally unstable spondylolisthesis. ${ }^{3}$ The combination of decompression and fusion shows a significantly better outcome than decompression alone. ${ }^{3}$

The Dynesys system (Zimmer, Winterthur, Switzerland) has been used as a semirigid stabilization device for the treatment of DLS since 1994. The pedicle screwbased system is intended to provide mobile stabilization, controlling motion in all 3 planes. It is designed to restore stability and treat painful degenerative conditions such as unstable degenerative disk disease and lumbar stenosis. Indications for this device are local lumbar pain and/or radicular pain attributed to instability with or without accompanying neurological deficit. Dynesys is also designed to stop further progression of minor deformities frequently associated with spinal stenosis, including degenerative spondylolisthesis and early degenerative scoliosis. 4,5 Biomechanical studies have shown that the Dynesys system allows for a nearly physiological spinal extension, whereas forward spinal flexion is comparable to that achieved after implantation of a fusion device. With this implant, lateral side bending is diminished up to $40 \%$, whereas rotation is not significantly reduced. 6,7 
TABLE 1. Age, Sex, Reason For Reintervention and Resurgery, and Time After Index Surgery in $n=8$ Cases

\begin{tabular}{|c|c|c|c|c|c|}
\hline $\begin{array}{l}\text { Case } \\
\text { Number }\end{array}$ & Age & Sex & Reason for Reintervention & Surgery & $\begin{array}{c}\text { Time After Index } \\
\text { Surgery }\end{array}$ \\
\hline 1 & 69 & $\mathrm{~F}$ & Spinal stenosis L2/3 with degenerative spondylolisthesis & $\begin{array}{l}\text { PLIF L2/3 } \\
\text { Decompression L2/3 }\end{array}$ & 9.4 \\
\hline 3 & 65 & $\mathrm{~F}$ & $\begin{array}{l}\text { Adjacent segment degeneration L3/4 with degenerative } \\
\text { spondylolisthesis }\end{array}$ & $\begin{array}{l}\text { Reinstrumentation, Dynesys } \\
\text { extension L2-5 }\end{array}$ & 6.4 \\
\hline 4 & 58 & $\mathrm{~F}$ & Adjacent segment degeneration L5/S1 & ALIF L5/S1 & 6 \\
\hline 5 & 45 & M & Adjacent segment degeneration L5/S1 with protrusion & ALIF L5/S1 & 3.5 \\
\hline 7 & 33 & $\mathrm{~F}$ & Adjacent segment degeneration L5/S1 & $\begin{array}{l}\text { ALIF } \mathrm{L} 4 / 5 \\
\text { disk prosthesis } \mathrm{L} 5 / \mathrm{S} 1\end{array}$ & 2.3 \\
\hline 8 & 39 & $\mathrm{~F}$ & Pedicle screw breakage L5 left & Prtial implant removal & 1.4 \\
\hline
\end{tabular}

ALIF indicates anterior lumbar interbody fusion; PLIF, posterior lumbar interbody fusion.

In theory, the preservation of some motion at the instrumented segment is intended to diminish adjacent segment degeneration. Short-term follow-up studies (up to $2 \mathrm{y}$ ) showed similar results of Dynesys compared with conventional fusion techniques regarding clinical outcome and perioperative and postoperative complications. ${ }^{5,8}$ However, long-term data are sparse.

This study presents a 7.2-year follow-up (range, 5-11.2 y) of DLS at the L4/5 level, treated with decompression and Dynesys stabilization.

\section{MATERIALS AND METHODS}

Between 2000 and 2006, 55 patients were treated using a monosegmental Dynesys system for DLS at the L4/5 level. Two experienced spine surgeons performed all surgeries using the same operative technique. All patients underwent decompression of bilateral recesses, undercutting laminotomies, and instrumentation.

Indication for implantation of the Dynesys device was determined on the basis of the preoperative clinical and radiologic evaluation. The preoperative evaluation included standard radiographs [anteroposterior (AP) and lateral], magnetic resonance imaging, and neurological and physical examination. Degenerative spondylolisthesis of $>1$ level or any prior lumbar surgery were contra- indications to implantation and excluded from this study. The minimum follow-up period was set to be 4 years.

Of the 55 patients treated during this period, there were 41 women $(74.5 \%)$ and 14 men $(25.5 \%)$ with a mean age at surgery of 60 years (range, $31-85 \mathrm{y}$ ). Thirty-nine patients $(71 \%$; 30 women, 9 men) with a mean age of 66 years (range, 40-91 y) were available for evaluation after a mean follow-up time of 7.2 years (range, 4.8-11.2 y). Sixteen patients $(29 \%)$ were lost to follow-up. Twelve $(22 \%)$ had died, $2(3.6 \%)$ could not be contacted, and 2 others $(3.6 \%)$ refused a visit because of immobility unrelated to spinal problems. The 2 patients unable to visit because of immobility reported over the telephone that they had no back or leg pain, but were not included in our follow-up.

\section{Clinical Outcome}

At follow-up investigation, standard neurological and physiological examination was performed. The need for pain medication was noted and any complications or additional operations directly related to the index surgery. For patient-based outcome measurement, a questionnaire described by Grob et $\mathrm{al}^{9}$ was used. The questionnaire assesses (1) changes in back and leg symptoms after surgery; (2) duration of improvement; (3) reoperations; (4) changes in work status; (5) quality of life and free-time

TABLE 2. Questionnaire According to Grob and Colleagues Concerning Remaining Back and Leg Pain, Improvement of Free-time Activities, and General Quality of Life

\begin{tabular}{lccccc}
\hline & Back Pain (\%) & Leg Pain (\%) & Work Status (\%) & Free Time (\%) & Quality of Life (\%) \\
\hline Much improved & 42 & 40 & 29 & 37 & 34 \\
Improved & 47 & 47 & 53 & 50 & 13 \\
No change & 3 & 8 & 5 & & \\
Worse & 8 & 5 & & & \\
Much improved & Surgery total & 50 & & & \\
Improved & 33 & & & \\
Helped a little & 8 & & & \\
No change & 5 & & & \\
Worse & Surgery again & & & \\
Yes & 82 & & \\
No & & &
\end{tabular}


TABLE 3. Spine Tango Scores at the Final Follow-Up

\begin{tabular}{|c|c|c|c|c|c|}
\hline "Spine Tango" Scores & Minimum & Maximum & Mean & Median & SD \\
\hline ODI score & 0.00 & 82.00 & 17.50 & 12.00 & 18.80 \\
\hline EQ-5D & 0.00 & 1.00 & 0.80 & 0.80 & 0.25 \\
\hline COMI & 0.00 & 9.60 & 3.00 & 2.90 & 2.60 \\
\hline
\end{tabular}

COMI indicates core outcome measures index; ODI, Oswestry Disability Index.

activities; and (6) global outcome. The questionnaire also asks the patient if they would undergo surgery again. In addition, patients were evaluated with the "Spine Tango" questionnaire, which includes the SF-36, EQ-5D, Oswestry Disability index (ODI), and Core Outcome Measures Index. These standardized questionnaires are established and validated instruments for the evaluation of postoperative outcomes. ${ }^{10}$

\section{Radiologic Outcome}

Radiographic assessment included AP, lateral, and functional radiographs. The follow-up images were compared with those taken immediately postoperatively. Two independent investigators (1 musculoskeletal radiologist and 1 orthopedic surgeon not involved in the original surgeries) analyzed all radiographs. Particular attention was paid to screw loosening and/or breakage. Any obvious motion of the screw on the functional radiographs, or any evidence of a typical radiolucent area around the screw was used to determine screw loosening. Degeneration at the operative level $(\mathrm{L} 4 / 5)$ and at the adjacent motion segments (L1/2, L2/3, L3/4, L5/S1) was analyzed using a linear 5-step scale (disk height not reduced, disk height $<25 \%$ reduced, disk height $25 \%-50 \%$ reduced, disk height $50 \%-75 \%$ reduced, disk height $>$ $75 \%$ reduced). To determine disk height reduction, the height of the disk at each motion segment was compared with the height of a healthy control disk. Motion at each segment was measured by comparing the angle of inclination and retrolisthesis for each segment in the functional radiographs. Potential progression of anterolisthesis in the treated segment was measured by comparing the "Sim index"11 from the immediate post- operative radiograph with that from the most recent follow-up. The Sim index quantifies the grade of spondylolisthesis in the lateral lumbar radiograph. It is calculated by dividing the length of the uncovered superior end plate of the caudal vertebral body by the full length of the same end plate. This quantifies the slip as a percentage where $0 \%$ is no slip, and $\geq 100 \%$ is a spondyloptosis.

\section{Statistical Analysis}

Wilcoxon signed-rank test was used for comparison of preoperative to postoperative continuous data. The level of significance was set to 0.05 throughout the study. All statistical analyses were conducted using SAS 9.1 (SAS Institute Inc., Cary, NC).

\section{RESULTS}

\section{Clinical Outcome}

Twenty-six percent of patients still required regular pain medicine for back pain, and an additional $7.8 \%$ used pain medicine occasionally for back pain. Sixty-seven percent of patients required no pain medication at all.

Fifteen patients $(38.5 \%)$ were retired from work at the time of the surgery. Twenty-one of the remaining 24 patients $(87.5 \%)$ returned to their previous level of work, leaving 3 patients $(12.5 \%)$ who did not return to their previous level of work because of persisting pain (2 patients, $8.3 \%$ ) or advancing age (1 patient, $4.2 \%)$.

Eight patients $(21 \%)$ required reoperation. Symptomatic adjacent segment disease was the main reason in 5 cases. In 1 case, the patient required a posterior lumbar interbody fusion of L2/3 for progressive degeneration at that level. There was 1 case of late-onset infection (7.3 y
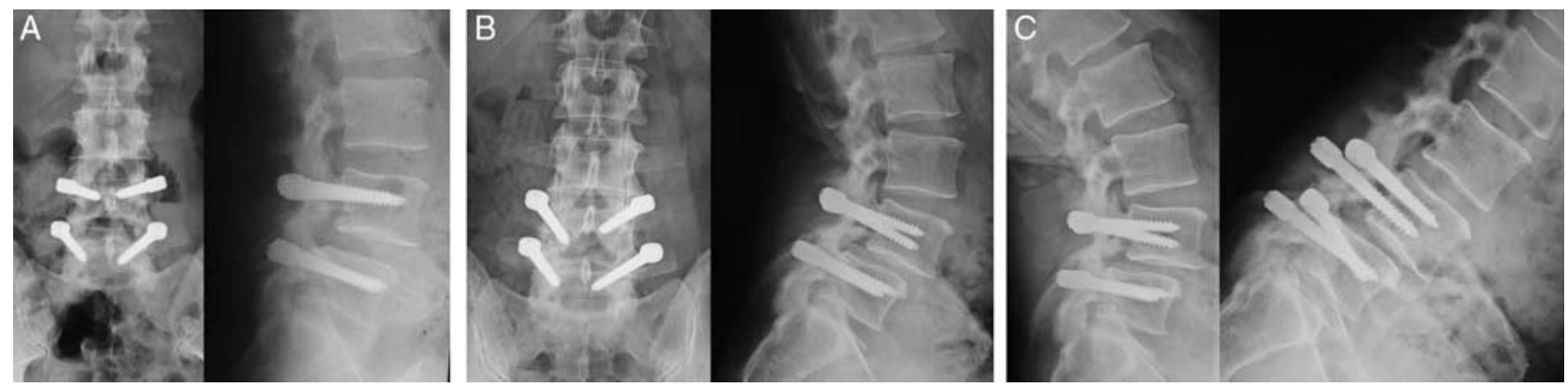

FIGURE 1. Case 1: 69 years, woman, 10-year FU, asymptomatic adjacent segment degeneration L3/4, visual analog scale leg pain: 0, visual analog scale back pain: 2, ODI: 20. A, Postoperative AP/lateral. B, Eleven-year FU AP/lateral: signs of adjacent segment degeneration L3/4. C, Eleven-year FU flexion/extension: <4 degrees range of motion at L4/5, adjacent segment degeneration L3/4. AP indicates anteroposterior; FU, follow-up; ODI, Oswestry Disability Index. 
TABLE 4. Average Motion in Extension/Flexion X-Rays in the Motion Segments L4/5 (Operated) and the Adjacent Motion Segments

\begin{tabular}{lccccc}
\hline & L4/5 & L5/S1 & L3/4 & L2/3 & L1/2 \\
\hline Median (deg.) & 2.2 & 8.3 & 7.4 & 8.3 & 7.8 \\
SD (deg.) & 3.0 & 7.0 & 5.8 & 5.4 & 5.1 \\
Maximum (deg.) & 11.0 & 28.0 & 24.0 & 21.0 & 16.0 \\
Minimum (deg.) & 0.0 & 0.0 & 0.0 & 0.0 & 0.0 \\
$<4^{\circ}$ & 29 & 10 & 9 & 8 & 12 \\
$>4^{\circ}$ & 10 & 29 & 30 & 31 & 27 \\
\hline
\end{tabular}

after index surgery) and 1 case of implant failure (1.4 y after surgery), both of whom were treated with partial implant removal (Table 1). Six of the 8 patients requiring reoperation went on to an overall satisfying result without further intervention. The other 2 patients continued to experience chronic pain (failed low back syndrome).

In the Grob questionnaire, $89 \%$ of patients stated that their back pain improved "very much" or "much" after surgery. Three percent reported no change in their pain, and $8 \%$ reported worsening back pain. Ten patients (25.6\%) experienced only a temporary improvement of back pain, with the average time of improvement being 3.6 years (range, $1-5 \mathrm{y}$; SD $1.6 \mathrm{y}$ ).

Similar results were seen with leg pain, which improved "very much" or "much" in $87 \%$ of patients. Eight percent of patients reported no change, and 5\% reported worse pain. Eleven of $39(28.2 \%)$ patients experienced only temporary improvement with the average time of improvement being 3.4 years (range, $1-5 \mathrm{y}$; SD $1.6 \mathrm{y}$ ).

Eighty-one percent of patients reported that their ability to work improved "very much" or "much." Thirteen percent reported no change and 5\% reported deterioration in their ability to work. The subjective quality of life improved "very much" or "much" in $84 \%$ of patients, no change was noted in $11 \%$, and $5 \%$ of the patients reported a "worse" quality of life after surgery.

Eighty-three percent of patients reported the operation "helped a lot" or "helped." Eight percent reported it "helped a little," 3\% reported no change, and 5\% reported a worsening after the intervention. Ninety-two percent of the patients would undergo the surgery again (Table 2).

\section{Spine Tango}

At most recent follow-up, the mean ODI was $17.5 \pm 18.8$, the mean EQ-5D Score was $0.8 \pm 0.25$, the mean Core Outcome Measures Index Score was $3.0 \pm 2.6$, and the mean SF-36 Physical Function Score was $45.0 \pm 10.3$ (Table 3 ).

TABLE 5. Disk Height as a Sign of Adjacent Segment Pathology in the Follow-Up X-Rays

\begin{tabular}{lccccc}
\hline & L4/5 & L5/S1 & L3/4 & L2/3 & L1/2 \\
\hline Disk height unchanged & 30 & 32 & 28 & 34 & 33 \\
Reduced disk height & 9 & 7 & 11 & 5 & 6 \\
\hline
\end{tabular}

$18.6 \%(29 / 156)$ of the adjacent segments showed signs of degeneration.

\section{Radiologic Outcome}

Nineteen percent $(29 / 156)$ of the adjacent segments showed signs of degeneration. Radiologic adjacent segment degeneration was most common in immediately adjacent segments L3/4 (11 patients, 28\%; Fig. 1) and L5/ S1 (7 patients, $18 \%$ ). L2/3 showed ongoing sings of degeneration in 5 patients $(13 \%)$, and L1/2 was affected in 6 patients $(15 \%)$. The stabilized segment L4/5 showed a mild progression of degeneration in 9 patients $(24 \%$; Table 4).

Range of motion at the instrumented segment was reduced. Twenty-nine segments $(73 \%)$ appeared to be fused on the functional radiographs with 16 segments $(41 \%)$ showing no motion, and 13 segments showing a flexion-extension range of $<4$ degrees. The maximal motion observed at the instrumented segment was 11 degrees (Table 5).

The Sim index, reflecting progression of vertebral AP slip, did not change in 32 of 39 patients $(82.1 \%)$ between the immediate postoperative $x$-ray and the follow-up.

Four screws in 3 patients showed radiologic signs of loosening without clinical symptoms. In 1 case, instability of the stabilized segment was found due to implant loosening. This patient was completely asymptomatic without leg or back pain (Fig. 2). In one of the 3 cases, 1 screw was observed to be broken. This patient opted for screw removal even though he reported no clinical symptoms related to the broken screw.

\section{DISCUSSION}

\section{Clinical Outcome}

This study presents long-term data from a single site treating a patient group with a clearly defined pathology using 1 type of surgery. Our data show that monosegmental instrumentation of a degenerative L4/5 spondylolisthesis with the Dynesys system, in combination with bilateral decompression, yielded good long-term results. Back and leg pain improved in $87 \%$ of patients, and $90 \%$ of patients were able to resume some form of work after surgery. Patient satisfaction was high, and $92 \%$ of the patients reported they would undergo the same operation again.

Current literature addressing long-term results of dynamic stabilization devices is sparse. Available data on short-term and mid-term results show mixed results. Schaeren and colleagues reported similar results to our study. They investigated 26 patients with degenerative spondylolisthesis at L3/4 or L4/5 with a 4-year follow-up. Stoll et $\mathrm{al}^{5}$ also showed significant improvement of ODI and pain scales in 83 patients stabilized with the Dynesys system for several different indications. Other authors describe less favorable results. Grob et $\mathrm{al}^{9}$ reported on a consecutive series of 50 patients with a 2-year follow-up after Dynesys instrumentation. Only $50 \%$ patients reported that the operation "helped" or "helped a lot." However, the study included various spinal pathologies, the indication for surgery was poorly defined, and monosegmental and mul- 

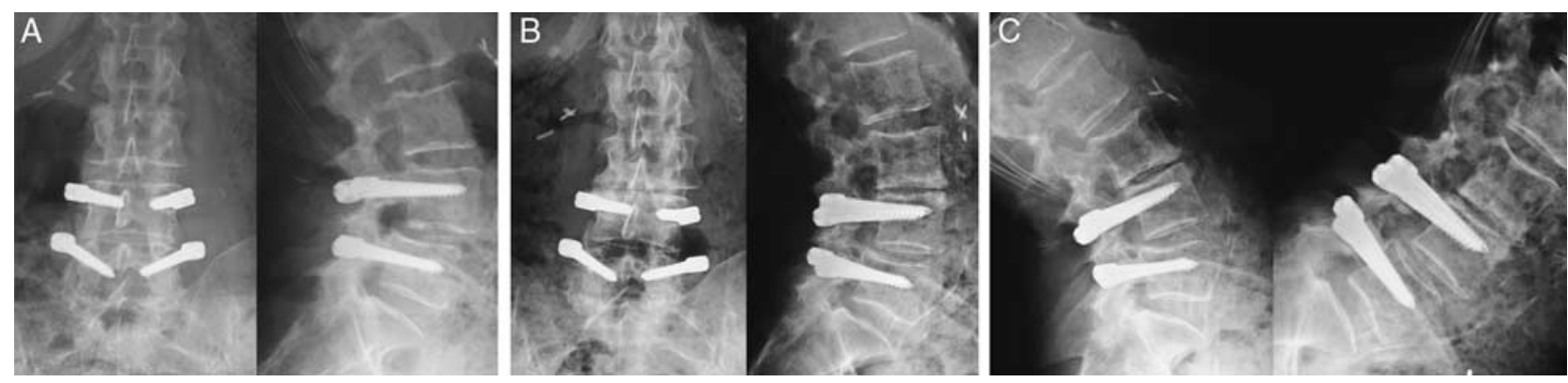

FIGURE 2. Case 2: 59 years, man, 5 -year FU, asymptomatic loosening of the $L 4$ screws with reinstability of L4/5. Visual analog scale leg pain and back pain: 0; ODI: 5. A, Postoperative AP/lateral. B, Five-year FU AP/lateral: screw loosening L4, increased anterolisthesis. C, Five-year FU flexion/extension: instability at L4/5. AP indicates anteroposterior; FU, follow-up; ODI, Oswestry Disability Index.

tisegmental stabilizations were not evaluated separately. Würgler-Hauri and colleagues showed a poor outcome in patient self-evaluation using the Coventry Scale. Patients reported an improvement of radicular pain but limited improvement of back pain after monosegmental and multisegmental Dynesys instrumentation in a short-term follow-up of 1 year. ${ }^{12}$ The authors concluded that decompression, rather than dynamic instrumentation, is responsible for improvement in their patients.

The value of decompression alone is arguable when reviewing data by Mannion and colleagues. They show that a "good" clinical outcome at 5 years after decompression alone can be achieved in $64.7 \%$ of patients with any degenerative spinal disease and compression of the neural elements. ${ }^{13}$

In a review of current literature, Carreon and colleagues showed that patients with degenerative spondylolisthesis generally benefit from stabilization compared with nonoperative treatment. Different kinds of fusion surgeries showed similar improvement in both ODI and SF-36 functional scores, supporting the widely established opinion that an unstable motion segment needs some kind of stabilization. ${ }^{3,14}$ Other authors also report good results after dorsal spondylodesis with autologous bone in degenerative spondylolisthesis at a follow-up of 2 years. ${ }^{15}$ Swan et $\mathrm{al}^{16}$ reported on the advantages of combined AP fusion (ODI, 14.6) over posterior instrumentation alone. Compared with detailed data in these studies, our results with dynamic stabilization show superior ODI and SF-36 scores.

\section{Revision Rate}

During the follow-up period, reoperations were required in 8 of 39 patients. The main reason for reintervention was symptomatic adjacent segment pathology in 6 patients at either L3/4 or L5/S1 (15.6\% of all patients). This is a relatively low percentage as the incidence of symptomatic adjacent segment pathology in the long term is reported to be between $15 \%$ and $35 \% .^{17-19}$

\section{Radiologic Outcome}

Our radiologic data show that dynamic stabilization with the Dynesys system did not lead to progression of vertebral slipping in the operated motion segment. Schaeren et $\mathrm{al}^{20}$ showed similar results in their study.
Progressive loss of disk height in the stabilized segment is rare. We found a limited mobility of $<4$ degrees in the stabilized segment in 29 of 39 patients, meaning almost $75 \%$ of stabilized segments become functionally fused over time. This could indicate that the Dynesys system works as a fusion device in a considerable percentage of cases and that clinically favorable results are not necessarily because of its potential motion-preserving character. This is supported by the fact that, radiologically, there was a progression of disk degeneration in the level above (L3/4) and below (L5/S1) the operated segment in $18 \%$ and $23 \%$, respectively. In contrast, we could not find a correlation between the rate of adjacent segment degeneration and remaining motion in the operated segment. This is in line with the results of a retrospective radiographic analysis by Cakir and colleagues where it was shown that Dynesys stabilization has no significant influence on the adjacent segment range of motion. Obviously preserving motion does not necessarily prevent adjacent segment disease, ${ }^{21,22}$ and the natural course of degenerative disease is difficult to predict.

\section{Limitations}

There are several limitations to our study. We do not have preoperative quality of life and pain data for all patients. Therefore, it is not possible to show the individual benefit of the operation in terms of improvement in the visual analog scale or SF-36 scores. However, the questionnaire according to Grob and colleagues is designed to assess the benefit of the operation retrospectively. Also, our study is a single-arm investigation without a control group, for example, patients treated with fusion or decompression alone. Nevertheless this study represents the largest patient cohort with degenerative spondylolisthesis treated in the described manner with the longest follow-up published so far.

\section{CONCLUSIONS}

Clinical long-term results after decompression and monosegmental instrumentation of degenerative L4/5 spondylolisthesis with the Dynesys system are overall good. The revision rate is comparable to other dorsal instrumentation devices. However, the majority of instrumented segments seem to functionally fuse over time. 
Nevertheless, the rate of radiologic and clinically symptomatic adjacent segment degeneration appears low. Dynesys stabilization of symptomatic L4/5 degenerative spondylolisthesis is a possible alternative to other stabilization devices.

\section{REFERENCES}

1. Lombardi JS, Wiltse LL, Reynolds J, et al. Treatment of degenerative spondylolisthesis. Spine (Phila Pa 1976). 1985;10:821-827.

2. Schwarzenbach O, Berlemann U. Dynamic posterior stabilization with the pedicle screw system DYNESYS(R). Oper Orthop Traumatol. 2010;22:545-557.

3. Kleinstueck FS, Fekete TF, Mannion AF, et al. To fuse or not to fuse in lumbar degenerative spondylolisthesis: do baseline symptoms help provide the answer? Eur Spine J. 2012;21:268-275.

4. Schwarzenbach O, Berlemann U, Stoll TM, et al. Posterior dynamic stabilization systems: DYNESYS. Orthop Clin North Am. 2005;36:363-372.

5. Stoll TM, Dubois G, Schwarzenbach O. The dynamic neutralization system for the spine: a multi-center study of a novel non-fusion system. Eur Spine J. 2002;11(suppl 2):S170-S178.

6. Schulte TL, Hurschler C, Haversath M, et al. The effect of dynamic, semi-rigid implants on the range of motion of lumbar motion segments after decompression. Eur Spine J. 2008;17:1057-1065.

7. Gedet P, Haschtmann D, Thistlethwaite PA, et al. Comparative biomechanical investigation of a modular dynamic lumbar stabilization system and the Dynesys system. Eur Spine J. 2009;18:1504-1511.

8. Schnake KJ, Schaeren S, Jeanneret B. Dynamic stabilization in addition to decompression for lumbar spinal stenosis with degenerative spondylolisthesis. Spine (Phila Pa 1976). 2006;31:442-449.

9. Grob D, Benini A, Junge A, et al. Clinical experience with the Dynesys semirigid fixation system for the lumbar spine: surgical and patient-oriented outcome in 50 cases after an average of 2 years. Spine (Phila Pa 1976). 2005;30:324-331.

10. Roder C, Chavanne A, Mannion AF, et al. SSE Spine Tangocontent, workflow, set-up. Eur Spine J. 2005;14:920-924. Available at http://www.eurospine.org.
11. Slim GP. Vertebral contour in spondylolisthesis. $\mathrm{Br} J$ Radiol. 1973;46:250-254.

12. Wurgler-Hauri CC, Kalbarczyk A, Wiesli M, et al. Dynamic neutralization of the lumbar spine after microsurgical decompression in acquired lumbar spinal stenosis and segmental instability. Spine (Phila Pa 1976). 2008;33:E66-E72.

13. Mannion AF, Denzler R, Dvorak J, et al. Five-year outcome of surgical decompression of the lumbar spine without fusion. Eur Spine J. 2010;19:1883-1891.

14. Carreon LY, Glassman SD, Howard J. Fusion and nonsurgical treatment for symptomatic lumbar degenerative disease: a systematic review of Oswestry Disability Index and MOS Short Form-36 outcomes. Spine J. 2008;8:747-755.

15. Jenis LG, Banco RJ, Kwon B. A prospective study of autologous growth factors (AGF) in lumbar interbody fusion. Spine J. 2006;6: 14-20.

16. Swan J, Hurwitz E, Malek F, et al. Surgical treatment for unstable low-grade isthmic spondylolisthesis in adults: a prospective controlled study of posterior instrumented fusion compared with combined anterior-posterior fusion. Spine J. 2006;6:606-614.

17. Rahm MD, Hall BB. Adjacent-segment degeneration after lumbar fusion with instrumentation: a retrospective study. $J$ Spinal Disord. 1996;9:392-400.

18. Throckmorton TW, Hilibrand AS, Mencio GA, et al. The impact of adjacent level disc degeneration on health status outcomes following lumbar fusion. Spine (Phila Pa 1976). 2003;28:2546-2550.

19. Ghiselli G, Wang JC, Bhatia NN, et al. Adjacent segment degeneration in the lumbar spine. J Bone Joint Surg Am. 2004;86A:1497-1503.

20. Schaeren S, Broger I, Jeanneret B. Minimum four-year follow-up of spinal stenosis with degenerative spondylolisthesis treated with decompression and dynamic stabilization. Spine (Phila Pa 1976). 2008;33:E636-E642.

21. Pellise F, Hernandez A, Vidal X, et al. Radiologic assessment of all unfused lumbar segments 7.5 years after instrumented posterior spinal fusion. Spine (Phila Pa 1976). 2007;32:574-579.

22. Cakir B, Carazzo C, Schmidt R, et al. Adjacent segment mobility after rigid and semirigid instrumentation of the lumbar spine. Spine (Phila Pa 1976). 2009;34:1287-1291. 